\title{
References
}

1 Morrell D, Cromartie E, Swift M. Mortality and cancer incidence in 263 patients with ataxia-telangiectasia. J Natl Cancer Inst 1986; 77: 89-92.

2 McGrath-Morrow SA, Gower WA, Rothblum-Oviatt C, et al. Evaluation and management of pulmonary disease in ataxia-telangiectasia. Pediatr Pulmonol 2010; 45: 847-859.

3 Bott L, Lebreton J, Thumerelle C, et al. Lung disease in ataxia-telangiectasia. Acta Paediatr 2007; 96: 1021-1024.

4 Schroeder SA, Zielen S. Infections of the respiratory system in patients with ataxia telangiectasia. Pediatr Pulmonol 2014; 49: 389-399.

5 Nowak-Wegrzyn A, Crawford TO, Winkelstein JA, et al. Immunodeficiency and infections in ataxia-telangiectasia. J Pediatr 2004; 144: 505-511.

6 Cystic Fibrosis Trust Microbiology Laboratory Standards Working Group. Laboratory standards for processing microbiological samples from people with cystic fibrosis. Bromley, Cystic Fibrosis Trust, 2010.

7 Haidopoulou K, Calder A, Jones A, et al. Bronchiectasis secondary to primary immunodeficiency in children: longitudinal changes in structure and function. Pediatr Pulmonol 2009; 44: 669-675.

8 Equi AC, Pike SE, Davies J, et al. Use of cough swabs in a cystic fibrosis clinic. Arch Dis Child 2001; 85: 438-439.

9 Chang AB, Newman RG, Carlin JB, et al. Subjective scoring of cough in children: parent-completed vs childcompleted diary cards vs an objective method. Eur Respir J 1998; 11: 462-466.

10 Berkhout MC, Rijntjes E, El Bouazzaoui LH, et al. Importance of bacteriology in upper airways of patients with Cystic Fibrosis. J Cyst Fibros 2013; 12: 525-529.

11 Bonestroo HJ, de Winter-de Groot KM, van der Ent CK, et al. Upper and lower airway cultures in children with cystic fibrosis: do not neglect the upper airways. J Cyst Fibros 2010; 9: 130-134.

12 Taylor CJ, McGaw J, Howden R, et al. Bacterial reservoirs in cystic fibrosis. Arch Dis Child 1990; 65: 175-177.

13 Mainz JG, Naehrlich L, Schien M, et al. Concordant genotype of upper and lower airways $P$. aeruginosa and S. aureus isolates in cystic fibrosis. Thorax 2009; 64: 535-540.

14 Staples ER, McDermott EM, Reiman A, et al. Immunodeficiency in ataxia telangiectasia is correlated strongly with the presence of two null mutations in the ataxia telangiectasia mutated gene. Clin Exp Immunol 2008; 153: 214-220.

15 van Ewijk B, van der Zalm M, Wolfs T, et al. Prevalence and impact of respiratory viral infections in young children with cystic fibrosis: prospective cohort study. Pediatrics 2008; 122: 1171-1176.

\section{Performance of the revised Geneva score in patients with a delayed suspicion of pulmonary embolism}

\author{
To the Editor:
}

Establishing a prompt diagnosis of acute pulmonary embolism is a diagnostic challenge, as the clinical presentation ranges from haemodynamic shock to very subtle symptoms mimicking those of other cardiovascular or pulmonary diseases [1]. This diverse presentation facilitates diagnostic delay and, consequently, also a delay in treatment initiation, which might be an important prognostic indicator for patients with acute pulmonary embolism [1]. The standard diagnostic algorithm for suspected acute pulmonary embolism consists of sequential pre-test probability determination, D-dimer testing and computed tomography pulmonary angiography (CTPA) [2]. The pre-test probability can be estimated using a validated clinical decision rule (CDR), such as the Wells score and the revised Geneva score (RGS) $[3,4]$. In addition to an excellent sensitivity and specificity, the main advantage of this diagnostic algorithm is that $20-30 \%$ of all patients with a clinical suspicion can be managed without CTPA, since an unlikely clinical probability in combination with a normal high-sensitive D-dimer test result has been shown to accurately rule out acute symptomatic pulmonary embolism [5]. The significance of the appropriate use of this diagnostic management strategy in patients with suspected pulmonary embolism has been highlighted by a prospective cohort study [6]. In patients with inappropriate diagnostic management, the diagnostic failure rate was $7.7 \%$, compared to $1.2 \%$ for those patients in whom pulmonary embolism was ruled out according to the strategy $(\mathrm{p}<0.001)$. Importantly, symptoms suggestive of pulmonary embolism that could also be ascribed to underlying cardiopulmonary diseases (e.g. heart failure or chronic lung disease) were identified as an important factor for diagnostic delay. Notably, uncertainty exists upon the validity of the recommended diagnostic algorithm in patients with a delayed clinical presentation, since both the pre-test probability and the D-dimer test result may be affected by the longer presence of blood clots in the pulmonary artery tree [7]. In addition, most diagnostic landmark studies only considered patients with an acute onset of symptoms $[3,8]$. A recent study reported that a delay in clinical presentation did not influence the safety of ruling out acute pulmonary embolism in the case of both an unlikely clinical 
probability by the Wells CDR and a normal D-dimer test [9]. The performance of the RGS, which is the second most frequently used and adequately validated CDR, has not yet been investigated in patients with a delayed presentation. Therefore, we aimed to assess the validity and efficiency of excluding pulmonary embolism in patients with a delayed clinical presentation based on the RGS combined with D-dimer testing.

The data of two prospective studies designed to investigate the diagnostic management of patients presenting with suspected pulmonary embolism were pooled; the Prometheus study and a sub-sample of patients included in the Christopher study [10,11]. Patients in the Christopher study were managed according to the Wells score combined with D-dimer testing and CTPA [12]. The performance of the RGS was assessed post hoc in a sub-population of this study [11]. The Prometheus study directly compared the performance of four CDRs, including the Wells score and the RGS, in 807 consecutive patients. For the present analysis, patients were classified as having an unlikely clinical probability if the revised Geneva score was $\leqslant 5$ points [10]. In these patients with an unlikely probability, we assessed the safety of high-sensitive D-dimer testing, including the VIDAS D-dimer assay (BioMerieux, Marcy L'Etoile, France) the Tinaquant assay (Roche Diagnostics, Basel, Switzerland), the STA-liatest D-di (Diagnostica Stago,Asnières sur Seine, France) and the Innovance D-dimer (Siemens, Munich, Germany), which was interpreted as normal at a cut-off level of $<500 \mu \mathrm{g} \cdot \mathrm{L}^{-1}$. CTPA was indicated in all other patients, and pulmonary embolism was confirmed if CTPA demonstrated at least one pulmonary arterial filling defect. All patients were followed for 3 months to assess the rate of venous thromboembolism during follow-up. For the present study, in consistence with previous literature [9], a delay in clinical presentation was defined as having complaints for $>7$ days before presentation. For the purpose of this analysis, we additionally used 10 days and 14 days as cut-offs for delay in clinical presentation. The efficiency of the RGS, defined as the percentage with an unlikely clinical probability for pulmonary embolism in combination with a normal D-dimer test result, was calculated and compared between patients with and without delay in presentation. False-negative rates were derived in both groups, defined as the incidence of symptomatic deep vein thrombosis or pulmonary embolism during follow-up in patients in whom pulmonary embolism was excluded on the basis of an unlikely RGS and a normal D-dimer test result. Differences in baseline characteristics and failure rate between groups with and without diagnostic delay were compared using Chi-Squared tests. SPSS version 20 (SPSS Inc., Chicago, IL, USA) was used to perform all analyses.

A total of 1107 patients were eligible for the present study. 19 patients were excluded from analyses since no data was recorded on the duration of complaints. Another 23 patients, derived from the Christopher population, were excluded because a D-dimer test was not performed as the Wells rule indicated pulmonary embolism was likely whilst the RGS categorised the patients post hoc as having an unlikely clinical probability. The characteristics of the remaining 1065 patients are depicted in table 1.

The percentage of patients with an unlikely RGS was comparable between patients with and without a delayed presentation $(70 \%$ versus $68 \%, \mathrm{p}=0.61)$. Similar rates were observed if patients were stratified using $\leqslant 10$ days $(69 \%$ versus $68 \%, p=0.83)$ and $\leqslant 14$ days $(68 \%$ versus $68 \%, p=0.99)$ as cut-off for diagnostic delay. In patients presenting within 7 days, pulmonary embolism was excluded based on a low RGS score in

\section{TABLE 1 Baseline demographics}

\begin{tabular}{|c|c|c|c|}
\hline & \multicolumn{2}{|c|}{ Delay in clinical presentation } & \multirow[t]{2}{*}{$p$-value } \\
\hline & $\leqslant 7$ days & $>7$ days & \\
\hline Subjects n & 869 & 196 & \\
\hline Age years & $51 \pm 17$ & $54 \pm 18$ & 0.009 \\
\hline Male & $342(39.4)$ & $79(40.3)$ & 0.806 \\
\hline Inpatient & 149 (17.2) & $22(11.2)$ & 0.041 \\
\hline COPD & $69(7.9)$ & $25(12.8)$ & 0.032 \\
\hline Heart failure & $40(4.6)$ & $12(6.1)$ & 0.373 \\
\hline \multicolumn{4}{|l|}{ VTE risk factors } \\
\hline $\begin{array}{l}\text { Immobilisation }>3 \text { days } \\
\text { or major surgery }<4 \text { weeks }\end{array}$ & $175(20.1)$ & $24(12.2)$ & 0.010 \\
\hline Active malignancy & 119 (13.7) & $22(11.2)$ & 0.357 \\
\hline History of VTE & $67(7.7)$ & $20(10.2)$ & 0.249 \\
\hline Oestrogen use & $127(14.6)$ & $22(11.2)$ & 0.217 \\
\hline
\end{tabular}

Data are presented as mean \pm SD or $n(\%)$, unless otherwise stated. COPD: chronic obstructive pulmonary disease; VTE: venous thromboembolism. 
combination with a normal D-dimer test result in 246 patients $(28 \%$ (95\% CI 25-31\%)) versus 40 patients $(20 \%(95 \%$ CI $15-27 \%))$ presenting after 7 days from the onset of symptoms $(\mathrm{p}=0.024)$. The false negative rate in patients presenting without delay was $0.4 \%$ (95\% CI $0.0-2.5 \%)$ compared to $0.0 \%$ (95\% CI 0.0-10\%) in patients with diagnostic delay $(\mathrm{p}=0.686)$. Similar failure rates were observed when using a cut-off $>10$ days ( $0.4 \%$ (95\% CI $0.0-2.5 \%)$ versus $0.0 \%(95 \%$ CI $0.0-12 \%), \mathrm{p}=0.708)$ and $>14$ days $(0.4 \%(95 \%$ CI $0.0-2.3 \%)$ versus $0.0 \%$ (95\% CI $0.0-17 \%), \mathrm{p}=0.767)$ for delay in presentation. A 65 -year-old male patient in whom the diagnostic algorithm had failed presented 1 day after the onset of acute, breathing-dependent chest pain and had subsegmental pulmonary embolism demonstrated by CTPA at baseline, despite having an unlikely clinical probability and a D-dimer test result of $482 \mu \mathrm{g} \cdot \mathrm{L}^{-1}$.

It should be stated that the present study represents a post hoc analysis that was not specifically powered to assess the safety of excluding pulmonary embolism in patients with and without delay in presentation. Nonetheless, our observations do not point towards a trend in an impaired efficiency and safety of this diagnostic algorithm among patients with diagnostic delay. A second limitation includes the fact that the specific symptoms and fluctuation of symptoms was not specified in the original studies. Also, it was not recorded whether patients had contacted other physicians prior to presenting to the study centres. Hence, our definition of diagnostic delay may have included doctor's delay in some cases, in addition to patients delay. Furthermore, the alternative diagnoses in patients in whom pulmonary embolism was ruled out were not specified. It cannot be ruled out that some of these patients did have chronic thromboembolic hypertension or chronic pulmonary embolism, although none of the patients were diagnosed with these diseases during follow-up.

Our observations indicate that it remains safe to use the RGS and a D-dimer test to rule out pulmonary embolism, regardless of the duration of symptoms before a suspicion of pulmonary embolism is raised. However, the efficiency of the RGS was significantly lower for patients with a delayed presentation, although the proportion in whom PE could be excluded without CTPA imaging remained $>20 \%$. with delayed suspicion http://ow.ly/sUcFk

Paul L. den Exter ${ }^{1,3}$, Pim van den Hoven ${ }^{1,3}$, Tom van der Hulle ${ }^{1}$, Inge C.M. Mos ${ }^{1}$, Renée A. Douma ${ }^{2}$, Josien van Es ${ }^{2}$, Menno V. Huisman ${ }^{1}$ and Frederikus A. Klok ${ }^{1}$

${ }^{1}$ Dept of Thrombosis and Hemostasis, Leiden University Medical Center, Leiden, and ${ }^{2}$ Dept of Vascular Medicine, Academic Medical Center, Amsterdam, The Netherlands. ${ }^{3}$ Both authors contributed equally.

Correspondence: P. L. den Exter, Dept of Thrombosis and Hemostasis, Leiden University Medical Center, Albinusdreef 2, P.O Box 9600, 2300 RC, Leiden, the Netherlands. E-mail: p.l.den_exter@lumc.nl

Received: Dec 092013 | Accepted after revision: Jan 082014 | First published online: Feb 132014

Conflict of interest: None declared.

\section{References}

1 Torbicki A, Perrier A, Konstantinides S, et al. Guidelines on the diagnosis and management of acute pulmonary embolism: the Task Force for the Diagnosis and Management of Acute Pulmonary Embolism of the European Society of Cardiology (ESC). Eur Heart J 2008; 29: 2276-2315.

2 Huisman MV, Klok FA. How I diagnose acute pulmonary embolism. Blood 2013; 121: 4443-4448.

3 Le Gal G, Righini M, Roy PM, et al. Prediction of pulmonary embolism in the emergency department: the revised Geneva score. Ann Intern Med 2006; 144: 165-171.

4 Wells PS, Anderson DR, Rodger M, et al. Derivation of a simple clinical model to categorize patients probability of pulmonary embolism: increasing the models utility with the SimpliRED D-dimer. Thromb Haemost 2000; 83: 416-420.

5 Pasha SM, Klok FA, Snoep JD, et al. Safety of excluding acute pulmonary embolism based on an unlikely clinical probability by the Wells rule and normal D-dimer concentration: a meta-analysis. Thromb Res 2010; 125: e123-e127.

6 Roy PM, Meyer G, Vielle B, et al. Appropriateness of diagnostic management and outcomes of suspected pulmonary embolism. Ann Intern Med 2006; 144: 157-164.

7 Bruinstroop E, van de Ree MA, Huisman MV. The use of D-dimer in specific clinical conditions: a narrative review. Eur J Intern Med 2009; 20: 441-446.

8 Wells PS, Anderson DR, Rodger M, et al. Excluding pulmonary embolism at the bedside without diagnostic imaging: management of patients with suspected pulmonary embolism presenting to the emergency department by using a simple clinical model and D-dimer. Ann Intern Med 2001; 135: 98-107.

9 den Exter PL, van Es J, Erkens PM, et al. Impact of delay in clinical presentation on the diagnostic management and prognosis of patients with suspected pulmonary embolism. Am J Respir Crit Care Med 2013; 187: 1369-1373.

10 Douma RA, Mos IC, Erkens PM, et al. Performance of 4 clinical decision rules in the diagnostic management of acute pulmonary embolism: a prospective cohort study. Ann Intern Med 2011; 154: 709-718. 
11 Klok FA, Kruisman E, Spaan J, et al. Comparison of the revised Geneva score with the Wells rule for assessing clinical probability of pulmonary embolism. J Thromb Haemost 2008; 6: 40-44.

12 van Belle A, Buller HR, Huisman MV, et al. Effectiveness of managing suspected pulmonary embolism using an algorithm combining clinical probability, D-dimer testing, and computed tomography. JAMA 2006; 295: 172-179.

\section{Assessment of ventriculo-arterial interaction in pulmonary arterial hypertension using wave intensity analysis}

To the Editor:

Wave intensity analysis (WIA) is a recently described haemodynamic analysis methodology which enables assessment of ventriculo-arterial interactions via time-domain analysis of pressure and flow waveforms [1]. It enables quantification of wave energy, separation of waves into forward and backward components, and estimation of wave speed [2]. To date, WIA has not been applied to the pulmonary arterial circulation in humans. As WIA offers a potential novel approach for the study of pulmonary haemodynamics, the present study sought to evaluate the feasibility of invasive WIA of the pulmonary circulation during right heart catheterisation (RHC).

The study was approved by the institutional review board (Sydney Local Health District Ethics Review Committee (RPA Zone)) and consent was obtained from seven controls (mean \pm SD age $69 \pm 9$ years, three females) and six patients (age 56 \pm 13 years, four females) with pulmonary arterial hypertension (PAH). Control subjects presented to the cardiac catheterisation laboratory for investigation of possible coronary artery disease. Following standard pulmonary haemodynamic measurements (including thermodilution cardiac output), a combined dual-tipped pressure and Doppler wire (ComboWire; Volcano, Rancho Cordova, CA, USA) was positioned distal to the origin of the right or left lower lobe pulmonary artery via a 6 Fr multipurpose catheter (Mach 1; Boston Scientific, Natick, MA, USA) under angiographic guidance. Pressure and flow measurements were acquired simultaneously at a sampling frequency of $1000 \mathrm{~Hz}$, once stable signals were obtained.

Data were processed offline using customised MatLab software (MathWorks, Natick, MA, USA). Signals were ensemble-averaged with timing gated to the ECG R-wave and smoothed using a Savitzky-Golay filter [3]. Intrinsic hardware processing delays (between pressure and flow signals) were corrected by shifting signals to achieve a linear pressure-flow (P-U) relationship in early systole [4]. Wave speed (c) was derived from the P-U loop method [2]:

$$
\mathrm{c}=(\mathrm{dP} / \mathrm{dU}) / \rho
$$

where $\mathrm{dP} / \mathrm{dU}$ is the slope of $\mathrm{P}-\mathrm{U}$ loop in early systole and $\rho$ is density of blood, assumed to be $1060 \mathrm{~kg} \cdot \mathrm{m}^{-3}$.

WIA was performed as previously described [3] with separation of waves into forward $\left(\mathrm{dI}_{+}\right)$and backward (dI_) wave intensities:

$$
\begin{aligned}
& \mathrm{dI}_{+}=+(\mathrm{dP} / \mathrm{dt}+\rho \mathrm{c} \times \mathrm{dU} / \mathrm{dt})^{2} / 4 \rho c \\
& \mathrm{dI}_{-}=-(\mathrm{dP} / \mathrm{dt}-\rho \mathrm{c} \times \mathrm{dU} / \mathrm{dt})^{2} / 4 \rho c
\end{aligned}
$$

Total wave energy for separated waves was calculated by integration of wave intensities over time. The magnitude of wave reflection (reflection coefficient) was calculated as the ratio of the total forward compression wave intensity to the total backward compression wave intensity [5]. The reflection distance (L) from the measurement site was estimated from the time delay between the peaks of the forward and backward compression waves, together with knowledge of wave speed:

$$
\mathrm{L}=\mathrm{c} \Delta \mathrm{t} / 2
$$

Results are presented as mean $\pm \mathrm{SD}$, unless otherwise stated. Differences between groups were compared using the Mann-Whitney U-test. Correlation was performed using Pearson's test. Significance was inferred at a two-sided $\mathrm{p}<0.05$. 